\title{
Study on static load test of modified and extended rigid frame bridge
}

\author{
NING Yu-feng ${ }^{1}$, YANG Qing-cheng ${ }^{1}$, SUN Wei-wei ${ }^{1 *}$, CHEN Shun-chao ${ }^{1}$ \\ ${ }^{1}$ College of Civil Engineering, Southwest Forestry University, Kunming Yunnan 650224
}

\begin{abstract}
In order to evaluate the mechanical condition and working performance of a rigid frame bridge after reconstruction and extension, the mechanical performance of the bridge was analyzed by static load test. Static load test is the test of stress at each section of main beam under the action of partial load and medium load. The results show that, under the action of each load, the check coefficient of strain and deflection at each measuring point of the box girder does not exceed the standard limit value 1 , the relative residual strain or deformation at each measuring point does not exceed the standard limit value $20 \%$, the stress condition of the tested section of the box girder is normal, and the structure is under the normal stress state under the action of the test load.
\end{abstract}

\section{Preface}

At present, more and more scholars have studied the bridge beam static load test, and some research results have been obtained. For example, Huang Jun and Li Shenglian et al. [2] carried out static load test on the trestle bridge of hydropower station by means of water tank loading. Dong Shuiying [3] conducted an experimental study on a 419.3T special vehicle passing through hollow slab bridge. Gu Yongjun [4] studied and analyzed the safe crossing of the bridge by special vehicles; Su Jianfeng, Xu Yufeng et al. [5] studied the safety assessment of wind power generation transport vehicles passing through simply supported beam Bridges. Li Desheng, Zhou Zhaohuan et al. [6] studied the load test of transporting wind turbine equipment across the bridge; Analysis of the Bearing capacity of box Girder bridge under special load by Liu Xiao-zhou. But at present, there are few literature about the research of load test for the reconstruction and extension of rigid frame bridge in China. Therefore, the research of this topic has application value.

\section{Project Overview}

The bridge is located at $\mathrm{K} 38+783$ of a national highway. The main bridge adopts a three-span prestressed continuous rigid frame system with the span layout of $60 \mathrm{~m}+100 \mathrm{~m}+60 \mathrm{~m}=220 \mathrm{~m}$. The transverse slope of the bridge deck is bi-directional $2 \%$, and the longitudinal slope of the bridge section is $2.4 \%$ and $2 \%$. The main girder of the bridge is a single-box single-chamber box section, C50 concrete casting, the top width of the box girder is $12.00 \mathrm{~m}$, the bottom width is $6.50 \mathrm{~m}$. The main pier body adopts double-column thin wall pier body, the external outline of the pier body is rectangular, C40 concrete casting. Bored pile foundation is adopted for foundation, U-shaped abutment is adopted for abutment, and 6 bored piles with diameter of $2.00 \mathrm{~m}$ are set under it. The design load of the bridge is highway -I class.

The finite element analysis software of Midas Civil bridge was used to calculate the calculation tool. The box girder and pier column were simulated by beam element. By applying static load to the model, the strain and displacement values of each measured point in each test section of the structure were obtained, and the bearing capacity of the bridge was compared with the measured values after the real bridge was loaded.

\section{Bridge beam static load test}

\subsection{Principle of static load test}

Static load test is mainly used to test the strain, displacement, residual strain and residual deformation of each control section measuring point of the upper main structure of the bridge [6], and comprehensively evaluate the performance of the main structure of the bridge. According to the calculation and analysis, six triaxial loading vehicles with a total weight of $400 \mathrm{kN}$ were used in the static load test of the bridge. The most unfavorable position of the influence line of internal force on the control section of the main structure was loaded so that the test load efficiency met the requirements of Equation 2-1 and the test load efficiency coefficient was between 0.85 and 1.05. The main technical indicators of the vehicle are shown in Figure 1.

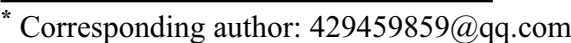



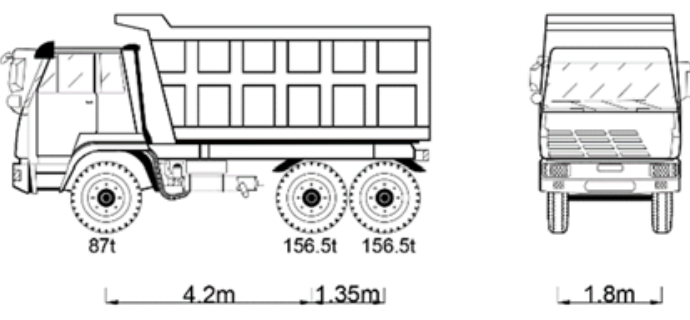

FIG. 1 Wheelbase diagram of test vehicle

Strain observation: in the long range control box girder section concrete setting concrete strain gauge $(100 \times 3 \mathrm{~mm}$, $120.1+/-0.1 \Omega$ resistance), using DH3819N static strain testing and analysis system for acquisition of each measuring point under the condition of the strain.

Deflection observation: a prism was arranged at the lower edge of the control section of the box beam, and the TCA1800 new Leica total station was used to measure the deflection of the measuring points under various working conditions.

\subsection{Test section and measuring point arrangement}

There are 5 test sections in total, namely, the maximum positive bending moment test sections JM1 and JM5 of the side span, the maximum negative bending moment test sections JM2 and JM4 of the fulcrum of the main span, and the maximum positive bending moment test section JM3 of the main span. The specific layout is shown in Figure 2.

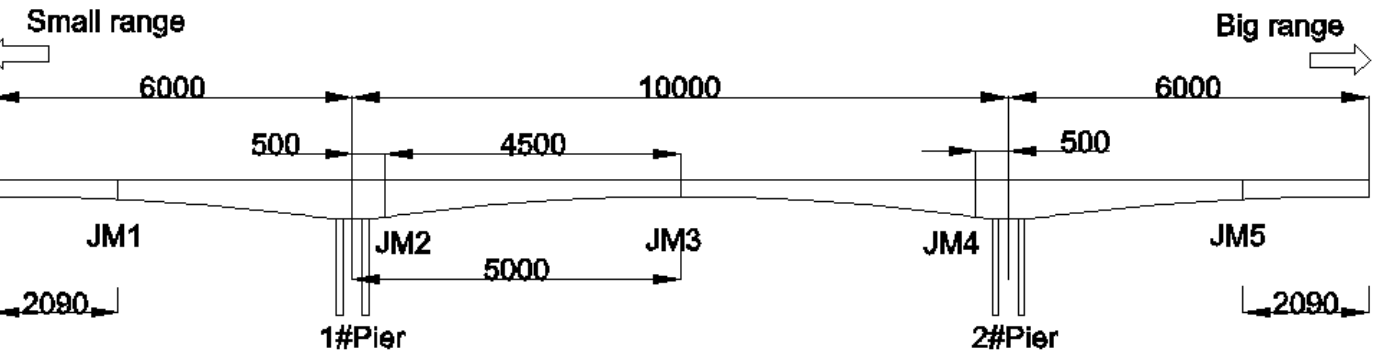

FIG. 2 Schematic Diagram of test section layout(cm)

The bridge JM1 and JM2, JM3, JM4, JM5 section strain measurement points are arranged in the box girder external (web plate and base plate), JM1, JM3, JM5 section are arranged seven points, three points, at the bottom of the left and right web 2 points each), and JM2, JM4 section are arranged in the 11 points, three points, at the bottom of the left and right web four points each), a total of 43 strain measuring point.

The measuring points of longitudinal deflection along the bridge are arranged on the left and right sides of the sidewalk, and the side span and middle span are divided into 4 equal parts. The test method adopts precision level and indium steel ruler to test.

\subsection{Test conditions and load arrangement}

The static load test of the bridge beam determines the test condition according to the most unfavorable force principle of the bridge structure. The longitudinal direction of the bridge is loaded according to the most unfavorable position. The transverse direction of the bridge is divided into medium load and partial load. The lateral arrangement of vehicles is shown in Figure $3 \sim 4$.

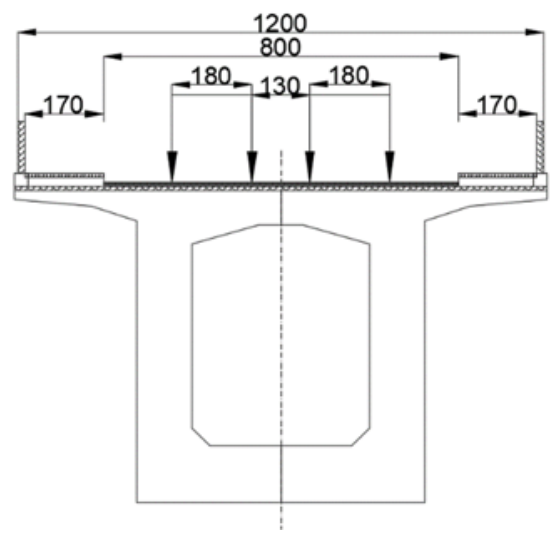

FIG. 3 Transverse mid-load layout of vehicles $(\mathrm{cm})$

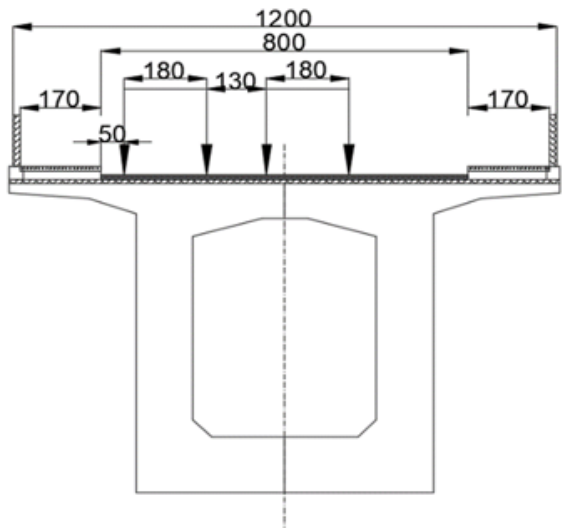

FIG. 4 Lateral load arrangement of vehicle $(\mathrm{cm})$ 


\subsection{Efficiency of static load test}

In the test load analysis, the two-lane load was considered, and the impact coefficient was considered in the design load, but the lateral reduction was not considered. The static load efficiency of the bridge was controlled by adjustment of loading position and loading tonnage, etc. $\eta_{\mathrm{q}}$ was between 0.85 and 1.05. See Table 1 for the value of static load efficiency of the bridge.

Table 1 Efficiency coefficient under static load

\begin{tabular}{|c|c|c|c|}
\hline $\begin{array}{c}\text { Load } \\
\text { condition }\end{array}$ & $\begin{array}{c}\boldsymbol{S}^{\prime} \cdot(\mathbf{1}+\boldsymbol{\mu}) / \\
\quad(\mathbf{k N} \cdot \mathbf{m})\end{array}$ & $\mathrm{S}_{\mathrm{s}} /(\mathbf{k} \mathbf{N} \cdot \mathbf{m})$ & $\eta_{\mathrm{q}}$ \\
\hline 1 & \multirow{2}{*}{11514.45} & \multirow{2}{*}{10829.50} & \multirow{2}{*}{0.94} \\
\hline 2 & & & \\
\hline 3 & \multirow{2}{*}{-35753.05} & \multirow{2}{*}{-37521.85} & \multirow{2}{*}{1.05} \\
\hline 4 & & & \\
\hline 5 & \multirow{2}{*}{11616.75} & \multirow{2}{*}{10599.32} & \multirow{2}{*}{0.91} \\
\hline 6 & & & \\
\hline 7 & \multirow{2}{*}{-35788.30} & \multirow{2}{*}{-37534.70} & \multirow{2}{*}{1.05} \\
\hline 8 & & & \\
\hline 9 & \multirow{2}{*}{11522.44} & \multirow{2}{*}{10845.61} & \multirow{2}{*}{0.94} \\
\hline 10 & & & \\
\hline
\end{tabular}

\subsection{Analysis of test results}

\subsubsection{Analysis of beam bottom strain test results}

Under static load test conditions from 1 to 10 , the strain test results and theoretical calculation values of some strain test sections are compared and analyzed as shown in Table 2.

Table 2 Comparison and analysis of static load strain results of section

\begin{tabular}{|c|c|c|c|c|c|c|}
\hline \multicolumn{2}{|l|}{ number } & \multirow{2}{*}{$\mathbf{S}_{\mathrm{e}} / \boldsymbol{\mu \varepsilon}$} & $\mathbf{S}_{\mathbf{p}} / \boldsymbol{\mu \varepsilon}$ & $\mathbf{S}_{\mathbf{s}} / \boldsymbol{\mu \varepsilon}$ & $\boldsymbol{\eta}$ & $\mathbf{S}_{\mathbf{p}}{ }^{\prime} / \%$ \\
\hline \multirow{2}{*}{1} & $3 \#$ & 37 & 1 & 56 & 0.66 & 2.63 \\
\cline { 2 - 7 } & $5 \#$ & 36 & 2 & 56 & 0.64 & 5.26 \\
\hline \multirow{2}{*}{2} & $3 \#$ & 35 & 1 & 56 & 0.63 & 2.78 \\
\cline { 2 - 7 } & $5 \#$ & 34 & 2 & 56 & 0.61 & 5.56 \\
\hline \multirow{2}{*}{3} & $4 \#$ & -25 & -4 & -37 & 0.68 & 13.79 \\
\cline { 2 - 7 } & $5 \#$ & -23 & -5 & -37 & 0.62 & 17.86 \\
\hline \multirow{3}{*}{4} & $4 \#$ & -26 & -2 & -37 & 0.70 & 7.14 \\
\cline { 2 - 7 } & $5 \#$ & -24 & -2 & -37 & 0.65 & 7.69 \\
\hline \multirow{3}{*}{5} & $3 \#$ & 33 & 2 & 66 & 0.50 & 5.71 \\
\cline { 2 - 7 } & $4 \#$ & 36 & 2 & 66 & 0.55 & 5.26 \\
\hline \multirow{3}{*}{6} & $3 \#$ & 37 & 1 & 66 & 0.56 & 2.63 \\
\cline { 2 - 7 } & $4 \#$ & 38 & 1 & 66 & 0.58 & 2.56 \\
\hline \multirow{2}{*}{7} & $4 \#$ & -22 & -1 & -37 & 0.59 & 4.35 \\
\cline { 2 - 7 } & $5 \#$ & -27 & -2 & -37 & 0.73 & 6.90 \\
\hline \multirow{5}{*}{8} & $4 \#$ & -24 & -1 & $-37 \mathrm{c}$ & 0.65 & 4.00 \\
\cline { 2 - 7 } & $5 \#$ & -26 & -2 & -37 & 0.70 & 7.14 \\
\cline { 2 - 7 } & $4 \#$ & 30 & 1 & 56 & 0.54 & 3.23 \\
\cline { 2 - 7 } & $5 \#$ & 34 & 2 & 56 & 0.61 & 5.56 \\
\cline { 2 - 7 } & $4 \#$ & 32 & 1 & 56 & 0.57 & 3.03 \\
\cline { 2 - 7 } & $5 \#$ & 31 & 2 & 56 & 0.55 & 6.06 \\
\hline \multirow{2}{*}{} & & & & & & \\
\hline
\end{tabular}

\subsubsection{Analysis of girder web strain test results}

The strain data of girder webs are shown in Table 3.

Table 3 Measured strain values of girder webs

\begin{tabular}{|c|c|c|c|}
\hline $\begin{array}{c}\text { Cross } \\
\text { section }\end{array}$ & point & $\begin{array}{c}\text { Distance } \\
\text { from beam } \\
\text { bottom (cm) }\end{array}$ & $\begin{array}{c}\text { Measured } \\
\text { elastic } \\
\text { strain (mm) }\end{array}$ \\
\hline \multirow{3}{*}{ JM1 } & $1 \#$ & 218 & -13 \\
\cline { 2 - 4 } & $2 \#$ & 18 & 28 \\
\cline { 2 - 4 } & $3 \#$ & 0 & 35 \\
\hline \multirow{3}{*}{ JM2 } & $1 \#$ & 522.5 & 14 \\
\cline { 2 - 4 } & $2 \#$ & 417.5 & 10 \\
\cline { 2 - 4 } & $3 \#$ & 100 & -18 \\
\hline \multirow{4}{*}{ JM3 } & $1 \#$ & 197 & -21 \\
\cline { 2 - 4 } & $2 \#$ & 30 & 35 \\
\cline { 2 - 4 } & $3 \#$ & 0 & 48 \\
\hline \multirow{3}{*}{ JM4 } & $1 \#$ & 522.5 & 17 \\
\cline { 2 - 4 } & $2 \#$ & 417.5 & 11 \\
\cline { 2 - 4 } & $3 \#$ & 100 & -18 \\
\hline \multirow{3}{*}{ JM5 } & $1 \#$ & 218 & -14 \\
\cline { 2 - 4 } & $2 \#$ & 18 & 29 \\
\cline { 2 - 4 } & $3 \#$ & 0 & 31 \\
\hline
\end{tabular}

\subsubsection{Analysis of deflection test results}

Under the test load of working conditions 1, 2, 5 and 6, the distribution comparison of measured deflection value and theoretical calculation value along the bridge longitudinal (including upstream and downstream) is shown in Figure $5 \sim 8$ below.

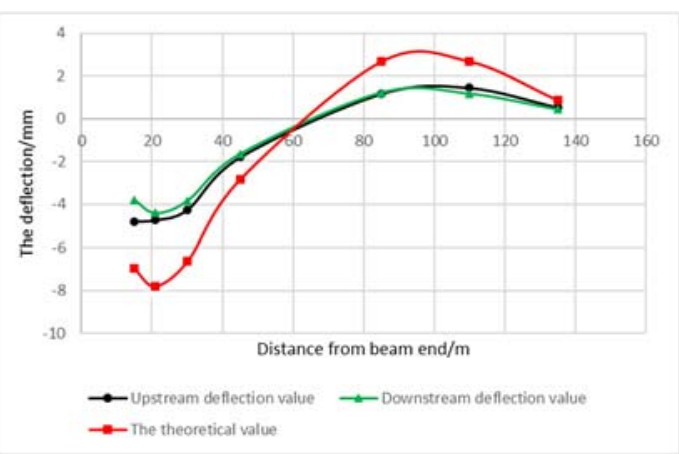

FIG. 5 Working Condition 1 Longitudinal deflection diagram (Partial load of JM1)

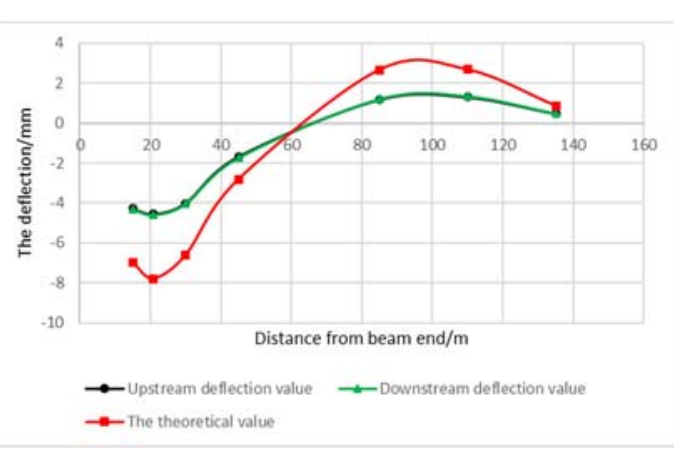

FIG. 6 Working condition 2 Longitudinal deflection diagram (JM1 intermediate load) 


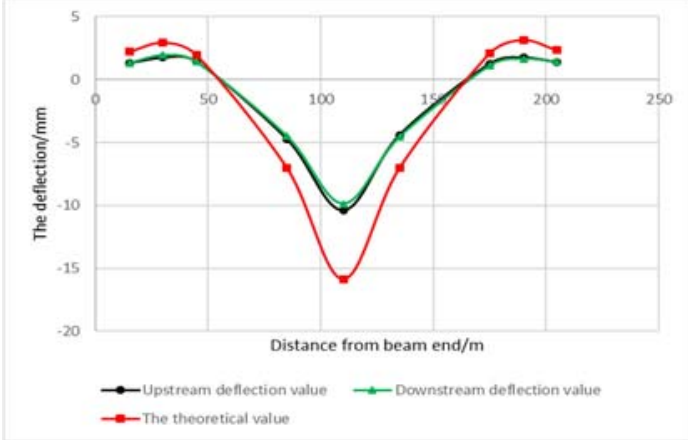

FIG. 7 Working Condition 5 Longitudinal deflection diagram (Partial load of JM3)

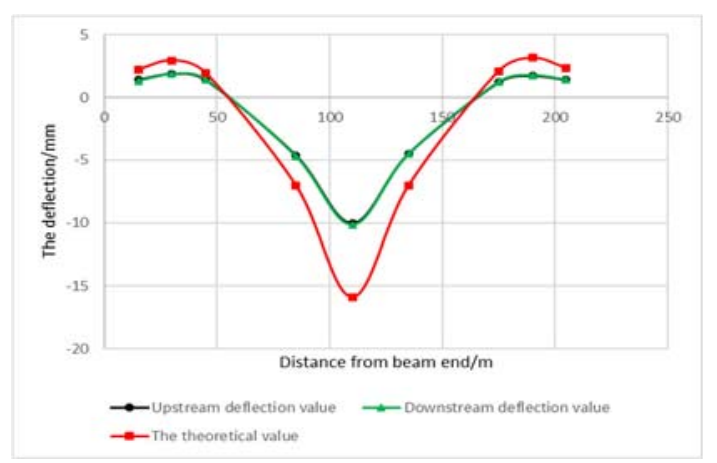

FIG. 8 Working condition 6 Longitudinal deflection diagram (JM3 intermediate load)

\section{Conclusion}

The measured strain, deflection results show that the box girder of each measuring point strain and deflection are not beyond the specification limit 1 calibration coefficient, relative residual strain or deformation of each measuring point did not exceed the standard limit by $20 \%$, test box girder section stress state without exception, the test section strain and deflection are not abnormal, the normal force of the structure, the overall carrying capacity of bridge structure meets the design load (road-grade I) normal use requirements.

\section{Reference}

1. Huang Jun, Li Liansheng, Lin Sheng,et al. Static load test for particularly loaded bridge in hydropower station[J]. Technology of Highway and Transport, 2010, (2): 52-55

2. Dong Shuiying. Static calculation and analysis of special load vehicle passing through hollow plate [J]. Technology of Highway and Transport (Applied Technology Edition), 2010, (6): 288-290

3. Gu Yongjun, Shi Ying. Research on reliability evaluation method and technical countermeasures for special load vehicle passing bridge [D]. Hang'zhou: Zhe jiang University of Technology, 2009

4. Su Jianfeng, Xu Yufeng. Research on special vehicle safety assessment for existing bridges[J]. Journal of China \& Foreign Highway, 2013, 33(4): 230-233
5. Li Desheng, Zhou Zhaohuan. Analysis of bridge load test with special vehicle load [J]. Urban Roads Bridges \& Flood Control, 2012, (1):55-57

6. Demeke B,Tommy H.T. Evaluation of dynamic loads on a skew box girder continuous bridge[J]. Engineering Structures, 2006, (29): 1052-1063

7. Ministry of Transport of the People's Republic of China, Specification for inspection and evaluation of load-bearing capacity of highway bridges: JTG/TJ212011 [S] 